\title{
8-Quinolinolates as Ligands for Luminescent Cyclometalated Iridium Complexes
}

Stefan Kappaun, ${ }^{\dagger}$ Stefan Sax, ${ }^{\dagger}$ Sabrina Eder, Kai C. Möller, Kerstin Waich, ${ }^{\dagger}$ Fabian Niedermair, ${ }^{\dagger}$ Robert Saf, ${ }^{\dagger}$ Kurt Mereiter, ${ }^{\S}$ Josemon Jacob, $^{++}$Klaus Müllen, ${ }^{++}$Emil J.W. List, ${ }^{\dagger}$ and Christian Slugove ${ }^{\dagger, *}$

${ }^{\dagger}$ Institute of Chemistry and Technology of Organic Materials (ICTOS), 'Institute of Chemical Technology of Inorganic Materials (ICTAS) and ${ }^{+}$Institute of Analytical Chemistry and Radiochemistry, Graz University of Technology, Stremayrgasse 16, A-8010 Graz, Austria,

${ }^{\ddagger}$ Institute of Solid State Physics, Graz University of Technology, Petersgasse 16, A-8010 Graz, Austria,

$\S$ Institute of Chemical Technologies and Analytics, Vienna University of Technology, Getreidemarkt 9/164SC, A-1060 Vienna, Austria, and

${ }^{++}$Max-Planck-Institute for Polymer Research, Ackermannweg 10, D-55128 Mainz, Germany

\section{Electronic Supplementary Information}

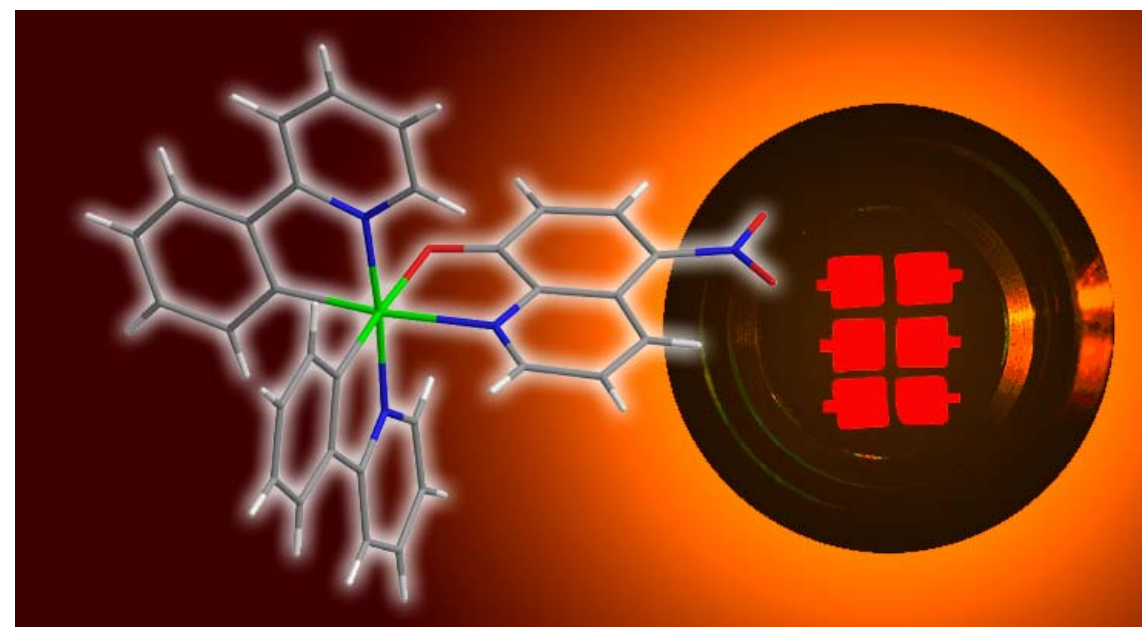

\section{Materials and Methods}

Unless otherwise noted, materials were obtained from commercial sources (Aldrich, Fluka or Lancaster) and were used without further purification. Solvents for reactions were freshly distilled over appropriate drying agents prior to use. Reactions were carried out under inert atmosphere of argon using standard Schlenk techniques.

${ }^{1} \mathrm{H}-\mathrm{NMR}$ spectra were recorded on a Varian INOVA $500 \mathrm{MHz}$ Spectrometer at $500 \mathrm{MHz}$, ${ }^{13} \mathrm{C}\left\{{ }^{1} \mathrm{H}\right\}$-NMR spectra were recorded at $125 \mathrm{MHz}$. Assignment of the peaks was done by DEPT and COSY NMR spectroscopy. Solvent residual peaks were used for referencing the NMR-spectra to the corresponding values given in literature. ${ }^{\mathrm{S} 1}$

\footnotetext{
${ }^{\mathrm{S} 1}$ Gottlieb, H. E.; Kotylar V.; Nudelman, A. J. Org. Chem., 1997, 62, 7512.
} 
UV-Visible absorption spectra were recorded on a Cary 50 Bio UV-Visible Spectrophotometer, fluorescence spectra on a Perkin Elmer Luminescence Spectrometer LS50B.

MALDI-TOF mass spectra were recorded on a Micromass TofSpec 2E. The instrument is equipped with a nitrogen laser (337 nm wavelength, operated at a frequency of $5 \mathrm{~Hz}$ ), and a time lag focusing unit. Spectra were taken in reflectron mode at an accelerating voltage of $+20 \mathrm{kV}$. Analysis of data was done with MassLynx 3.4 (Micromass, Manchester, UK). Samples were dissolved in THF $(1 \mathrm{mg} / \mathrm{mL})$. Dithranol or retinoic acid was used as matrix (10 $\mathrm{mg} / \mathrm{mL}$ in THF). Solutions were mixed in the cap of a microtube in the ratio of $1 \mu \mathrm{L}: 10 \mu \mathrm{L}$. $0.5 \mu \mathrm{L}$ of the resulting mixture was spotted onto the target and air dried.

Combined DSC/TGA measurements were performed with a Netzsch STA 449C equipped with a Quadrupole MS QMS 403C. The thermal properties were determined in aluminum pans with a heat rate of $10^{\circ} \mathrm{C} / \mathrm{min}$ in a helium flow of approximately $50 \mathrm{~mL} / \mathrm{min}$.

Luminescence excitation and emission spectra of thin films were acquired in air and while flushing coated capillaries with nitrogen with a Fluorescence Spectrometer Perkin Elmer LS50B. All spectra were recorded in the fluorescence measuring mode. Phase angle measurements were performed using a dual-phase lock-in amplifier (DSP 830, Stanford Research Inc.) for sine-wave modulation of the LED at a frequency of 5, 10 and $15 \mathrm{kHz}$ and for detection. The optical system consisted of a blue LED (130 mcd, $\lambda_{\max }=395-400 \mathrm{~nm}, 5 \mathrm{~mm}$, $3.1 \mathrm{~V}$, Lumitronix, Jungingen, Germany), a bifurcated glass fiber bundle of $2 \mathrm{~mm}$ i. d. and a red-sensitive PMT module (H5701-02, Hamamatsu; Herrsching, Germany) equipped with a long-pass filter (OG 550, Schott, Mainz, Germany). The measurements were carried out in air and under nitrogen atmosphere. The apparent lifetime was calculated according to the following equation with the phase shift $\phi$, modulation frequency $v$ and lifetime $\tau$.

$$
\tan \Delta \phi=2 \pi \nu \tau \text {. }
$$

Electrochemical measurements were carried out with an AUTOLAB PGSTAT100 high voltage potentiostat/galvanostat, manufactured by Eco Chemie B.V., Utrecht, Netherlands. For control, data acquisition and analysis the provided software, General Purpose Electrochemical System (GPES), version 4.9.004, developed by Eco Chemie, was used. The measurements were performed with a standard scan rate of $0.1 \mathrm{~V} / \mathrm{s}$ in $\mathrm{CH}_{2} \mathrm{Cl}_{2} / \mathrm{Bu}_{4} \mathrm{ClO}_{4}$ as electrolyte, using a three electrode cell with a platinum disc electrode as working electrode, a platinum counter electrode, and a silver pseudo-reference electrode which was calibrated with ferrocene/ferrocenium as internal standard. Due to the poor conductivity of the solvent / supporting electrolyte a positive feedback IR compensation was used to eliminate the IR drop between reference and working electrode.

Organic light emitting devices (OLEDs) were fabricated on glass substrates with transparent indium-tin-oxide (ITO) anodes in a commonly used sandwich structure (ITO/PEDOT:PSS/host+complex/Ca/Al). First, the samples were cleaned in an ultrasonic bath using a series of different organic solvents. Subsequently, they were etched for $10 \mathrm{~min}$ in an oxygen plasma. Then poly(3,4-ethylenedioxythiophene):poly(styrenesulfonate) (PEDOT:PSS) was applied by spin-coating under ambient conditions (approximate thickness $50 \mathrm{~nm}$ ). The samples were annealed in a vacuum oven at a temperature of $160^{\circ} \mathrm{C}$ for at least $20 \mathrm{~min}$, followed by cooling to room temperature over $40 \mathrm{~min}$. The polymer-metal complex blends were spin-coated from $\mathrm{CHCl}_{3}$ solutions. Ca top electrodes and an $\mathrm{Al}$ capping layer were evaporated in an evaporation chamber located in an argon glove box (exposure to air during the evaporation processes was kept at a minimum). Devices were tested in argon atmosphere to avoid oxidation. Device characteristics were recorded using a customized setup. While current/voltage characteristics were measured using a Keithley 236 Source Measure Unit, a photodiode mounted in an integrating sphere and connected to a Keithley 181 Nanovoltmeter was used to measure the emission intensity. To obtain luminance values (for devices operated in cw-mode), the set-up was calibrated using a Luminance Meter LS100 
(Minolta) near the maximum emission intensity. Electroluminescence spectra were obtained with a DB401-UV CCD detector (Andor) mounted on an Oriel 77400 grid spectrometer. The spectral sensitivity was calibrated with an Ocean Optics LS-1-CAL tungsten-halogen lamp.

\section{Synthesis}

Preparation of di- $\mu$-chlorotetrakis $\left(\kappa^{2}\left(C^{2}, N\right)\right.$-2-phenylpyridine)diiridium(III). ${ }^{\mathrm{S} 2}$
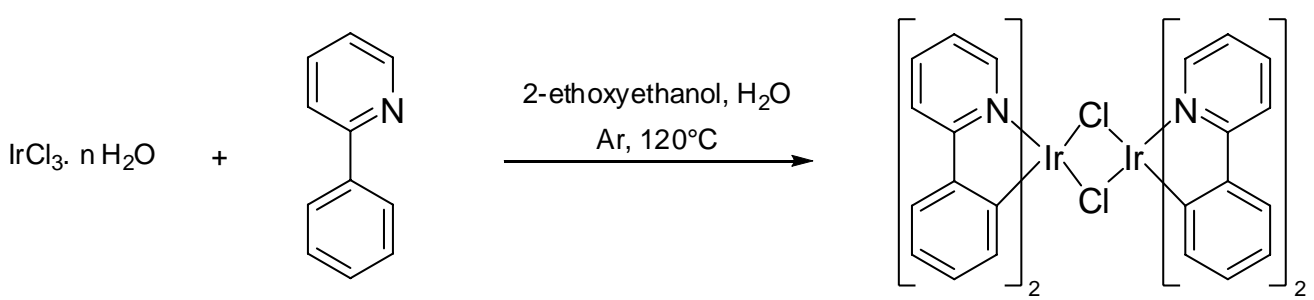

$\mathrm{IrCl}_{3} \cdot \mathrm{H}_{2} \mathrm{O}(0.2123 \mathrm{~g}, 0.711 \mathrm{mmol})$ was suspended in a mixture of degassed 2-ethoxyethanol (6 $\mathrm{mL})$ and water $(2 \mathrm{~mL})$ under inert atmosphere of argon. After the addition of 2-phenylpyridine $(270 \mu \mathrm{L}, 1.889 \mathrm{mmol})$ the dark brown reaction mixture was stirred for $20 \mathrm{~h}$ at $120^{\circ} \mathrm{C}$. The suspension turned greenish during that time. The reaction mixture was cooled to room temperature and the solvent was removed in vacuo. Washing the residue with methanol gave rise to a yellow-greenish powder. Yield: $202.5 \mathrm{mg}(53.1 \%)$.

Because of the decreased solubility in acetone- $\mathrm{d}_{6}$ or $\mathrm{CDCl}_{3}, \mathrm{DMSO}-\mathrm{d}_{6}$ was used as the solvent for NMR spectroscopy. Because of the coordinative properties of DMSO- $\mathrm{d}_{6}$, the dimer was cleaved and spectra listed below represent the structure shown on the right. Spectra of the dimer in noncoordinating solvents can be found in literature. ${ }^{\mathrm{S} 2}$

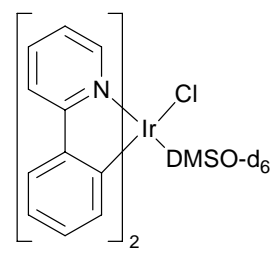

${ }^{1} \mathrm{H}-\mathrm{NMR}\left(\delta, 20^{\circ} \mathrm{C}\right.$, DMSO-d $\left.6,500 \mathrm{MHz}\right): 9.81\left(\mathrm{dd}, 2 \mathrm{H},{ }^{3} J_{\mathrm{HH}}=5.1 \mathrm{~Hz},{ }^{4} J_{\mathrm{HH}}=0.9 \mathrm{~Hz}, \mathrm{py}^{6}\right)$, $9.53\left(\mathrm{dd}, 2 \mathrm{H},{ }^{3} J_{H H}=5.1 \mathrm{~Hz},{ }^{4} J_{H H}=0.9 \mathrm{~Hz}, \mathrm{py}^{6}\right), 8.26\left(\mathrm{~d}, 2 \mathrm{H},{ }^{3} J_{\mathrm{HH}}=7.8 \mathrm{~Hz}, \mathrm{py}{ }^{3}\right), 8.18(\mathrm{~d}, 2 \mathrm{H}$, $\left.{ }^{3} J_{H H}=7.8 \mathrm{~Hz}, \mathrm{py}^{3}\right), 8.09\left(\mathrm{ddd}, 2 \mathrm{H}, 2 \mathrm{x}^{3} J_{\mathrm{HH}}=7.6 \mathrm{~Hz},{ }^{4} J_{H H}=1.5 \mathrm{~Hz}, \mathrm{py}^{4}\right), 8.01(\mathrm{ddd}, 2 \mathrm{H}, 2 \mathrm{x}$ $\left.{ }^{3} J_{H H}=7.6 \mathrm{~Hz},{ }^{4} J_{H H}=1.5 \mathrm{~Hz}, \mathrm{py}^{4}\right), 7.79\left(\mathrm{dd}, 2 \mathrm{H},{ }^{3} J_{\mathrm{HH}}=6.8 \mathrm{~Hz},{ }^{4} J_{\mathrm{HH}}=0.9 \mathrm{~Hz}, \mathrm{ph}^{6}\right), 7.73(\mathrm{dd}$, $\left.2 \mathrm{H},{ }^{3} J_{\mathrm{HH}}=7.0 \mathrm{~Hz},{ }^{4} J_{\mathrm{HH}}=0.8 \mathrm{~Hz}, \mathrm{ph}^{6}\right), 7.57\left(\mathrm{ddd}, 2 \mathrm{H},{ }^{3} J_{\mathrm{HH}}=7.3 \mathrm{~Hz},{ }^{3} J_{\mathrm{HH}}=5.8 \mathrm{~Hz},{ }^{4} J_{\mathrm{HH}}=\right.$ $\left.1.5 \mathrm{~Hz}, \mathrm{py}^{5}\right), 7.45\left(\mathrm{ddd}, 2 \mathrm{H},{ }^{3} J_{\mathrm{HH}}=7.3 \mathrm{~Hz},{ }^{3} J_{\mathrm{HH}}=5.8 \mathrm{~Hz},{ }^{4} J_{\mathrm{HH}}=1.5 \mathrm{~Hz}, \mathrm{py}^{5}\right), 6.89$ (ddd, $2 \mathrm{H}$, $\left.2 \mathrm{x}{ }^{3} J_{H H}=7.3 \mathrm{~Hz},{ }^{4} J_{H H}=1.0 \mathrm{~Hz}, \mathrm{ph}^{5}\right), 6.83\left(\mathrm{ddd}, 2 \mathrm{H}, 2 \mathrm{x}^{3} J_{H H}=7.3 \mathrm{~Hz},{ }^{4} J_{H H}=1.0 \mathrm{~Hz}, \mathrm{ph}^{5}\right)$, $6.76\left(\mathrm{ddd}, 2 \mathrm{H}, 2 \mathrm{x}^{3} J_{H H}=7.3 \mathrm{~Hz},{ }^{4} J_{H H}=1.2 \mathrm{~Hz}, \mathrm{ph}^{4}\right), 6.69\left(\mathrm{ddd}, 2 \mathrm{H}, 2 \mathrm{x}{ }^{3} J_{H H}=7.3 \mathrm{~Hz},{ }^{4} J_{H H}=\right.$ $\left.1.2 \mathrm{~Hz}, \mathrm{ph}^{4}\right), 6.25\left(\mathrm{dd}, 2 \mathrm{H},{ }^{3} J_{\mathrm{HH}}=6.8 \mathrm{~Hz},{ }^{4} J_{\mathrm{HH}}=0.7 \mathrm{~Hz}, \mathrm{ph}^{3}\right), 5.66\left(\mathrm{dd}, 2 \mathrm{H},{ }^{3} J_{H H}=6.8 \mathrm{~Hz}\right.$, $\left.{ }^{4} J_{H H}=0.7 \mathrm{~Hz}, \mathrm{ph}^{3}\right)$.

${ }^{13} \mathrm{C}-\mathrm{NMR}\left(\delta, 20^{\circ} \mathrm{C}\right.$, DMSO-d $\left.6,125 \mathrm{MHz}\right): 167.3,167.0\left(4 \mathrm{C}, \mathrm{py}^{2}\right), 152.1,150.6\left(4 \mathrm{C}, \mathrm{py}^{6}\right)$, $152.0,145.4,143.8,143.2\left(8 \mathrm{C}, \mathrm{ph}^{1,2}\right), 139.2,138.2,131.3,129.9,129.7,129.0,124.8,123.8$, $123.6,122.8,122.3,122.0,120.1,119.5\left(28 \mathrm{C}, \mathrm{py}^{3,4,5} \mathrm{ph}^{3,4,5,6}\right)$.

\footnotetext{
S2 (a) Sprouse, S.; King, K. A.; Spellane, P. J.; Watts, R. J. J. Am. Chem. Soc., 1984, 106, 6647. (b) Nonoyama,
} M. Bull. Chem. Soc. Jpn. 1974, 47, 767. 
Preparation of $(O C-6-42)-b i s\left(\kappa^{2}\left(C^{2}, N\right)\right.$-2-phenylpyridine $)\left(\kappa^{2}(N, O)-8\right.$-quinolinolate $)$ iridium(III) (1)


Di- $\mu$-chlorotetrakis $\left(\kappa^{2}\left(C^{2}, N\right)\right.$-2-phenylpyridine $)$ diiridium(III) $(0.0403 \mathrm{~g}, 0.038 \mathrm{mmol}, 1$ eq. $)$ and 8-hydroxyquinoline $(0.0126 \mathrm{~g}, 0.087 \mathrm{mmol}, 2.3 \mathrm{eq}$.) were suspended in a solvent-mixture of degassed $\mathrm{CH}_{2} \mathrm{Cl}_{2} / \mathrm{EtOH} / \mathrm{Et}_{3} \mathrm{~N}(1000 \mu \mathrm{L} / 1000 \mu \mathrm{L} / 300 \mu \mathrm{L})$ under inert atmosphere of argon. The suspension was refluxed at $80^{\circ} \mathrm{C}$ for 20 hours by which the mixture turned yellow-orange. The solvent was removed in vacuo and the residue was washed with methanol. Filtering off the yellow-orange precipitate gave the product in pure form. Yield $37.9 \mathrm{mg}(78.2 \%)$. $\mathrm{R}_{\mathrm{f}}($ acetone $)=0.86$.

${ }^{1} \mathrm{H}-\mathrm{NMR}\left(\delta, 20^{\circ} \mathrm{C}\right.$, DMSO-d $\left.6,500 \mathrm{MHz}\right): 8.55\left(\mathrm{~d}, 1 \mathrm{H},{ }^{3} J_{\mathrm{HH}}=5.8 \mathrm{~Hz}, \mathrm{py}^{6}\right), 8.17,8.13(2 \mathrm{xd}$, $\left.2 \mathrm{H},{ }^{3} J_{H H}=8.5 \mathrm{~Hz}, \mathrm{~d}, 1 \mathrm{H},{ }^{3} J_{\mathrm{HH}}=8.3 \mathrm{~Hz}, \mathrm{py}^{3}, \mathrm{q}^{4}\right), 7.86-7.78,7.36-7.32\left(\mathrm{~m}, 4 \mathrm{H}, \mathrm{m}, 2 \mathrm{H}, \mathrm{py}^{4}\right.$, $\left.\mathrm{ph}^{6}, \mathrm{q}^{3,5}\right), 7.58,7.50\left(\mathrm{dd}, 1 \mathrm{H},{ }^{3} J_{H H}=4.6 \mathrm{~Hz},{ }^{4} J_{H H}=1.5 \mathrm{~Hz}, \mathrm{~d}, 1 \mathrm{H},{ }^{3} J_{H H}=4.9 \mathrm{~Hz}, \mathrm{py}^{6}, \mathrm{q}^{2}\right), 7.26$ $-7.23\left(\mathrm{ddd}, 1 \mathrm{H}, 2 \mathrm{x}{ }^{3} J_{H H}=\right.$ ca. $\left.7 \mathrm{~Hz},{ }^{4} J_{H H}=1.2 \mathrm{~Hz}, \mathrm{py}^{5}\right), 7.09-7.06\left(\mathrm{ddd}, 1 \mathrm{H}, 2 \mathrm{x}{ }^{3} J_{H H}=\right.$ ca. $\left.6.5 \mathrm{~Hz},{ }^{4} J_{H H}=1.2 \mathrm{~Hz}, \mathrm{py}^{5}\right), 6.91-6.69\left(\mathrm{~m}, 6 \mathrm{H}, \mathrm{ph}^{4}, \mathrm{ph}^{5}, \mathrm{q}^{6,7}\right), 6.33\left(\mathrm{~d}, 1 \mathrm{H},{ }^{3} J_{\mathrm{HH}}=7.0 \mathrm{~Hz}\right.$, $\left.\mathrm{ph}^{3}\right)$ and $6.13\left(\mathrm{~d}, 1 \mathrm{H},{ }^{3} J_{\mathrm{HH}}=7.1 \mathrm{~Hz}, \mathrm{ph}^{3}\right)$.

${ }^{13} \mathrm{C}-\mathrm{NMR}\left(\delta, 20^{\circ} \mathrm{C}\right.$, DMSO-d $\left.6,125 \mathrm{MHz}\right): 169.3,168.0\left(2 \mathrm{C}, \mathrm{py}^{2}\right), 167.3\left(1 \mathrm{C}, \mathrm{q}^{8}\right), 152.0$, $150.5\left(2 \mathrm{C}, \mathrm{ph}^{2}\right), 147.8,147.7,145.4\left(3 \mathrm{C}, \mathrm{py}^{6}, \mathrm{q}^{2}\right), 144.7,144.5,143.5\left(3 \mathrm{C}, \mathrm{ph}^{1}, \mathrm{q}^{\mathrm{8a}}\right), 137.6$, $137.6,137.1,132.1,131.9,130.3,129.5,128.8,124.5,124.1,122.9,122.6,122.2,120.7$, $120.5, \quad 119.2, \quad 119.0\left(17 \mathrm{C}, \mathrm{ph}^{3,4,5,6}, \quad \mathrm{py}^{3,4,5}, \mathrm{q}^{3,4,6}\right), 131.4\left(1 \mathrm{C}, \mathrm{q}^{4 \mathrm{a}}\right), 116.0\left(1 \mathrm{C}, \mathrm{q}^{5}\right)$ and $109.9\left(1 \mathrm{C}, \mathrm{q}^{7}\right)$.

MALDI MS (m/z): [ $\left.\mathrm{C}_{31} \mathrm{H}_{22} \mathrm{IrN}_{3} \mathrm{O}\right] 643.1313$ (calcd 643.1369). 


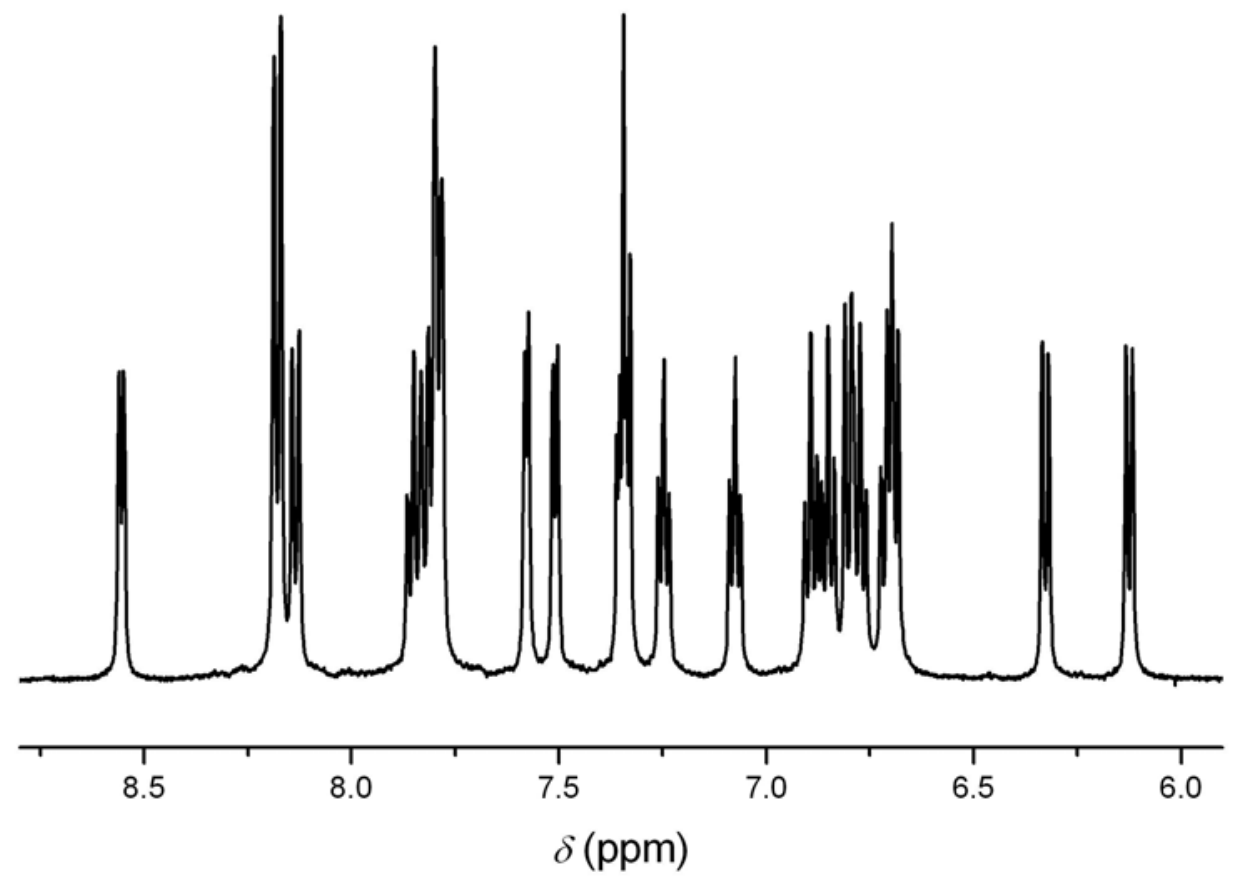

Figure S1. ${ }^{1} \mathrm{H}-\mathrm{NMR}$ spectrum of $\mathbf{1}$ in DMSO-d 6



Figure S2. Calculated (above) and measured (below) isotope pattern of $\mathbf{1}$ 


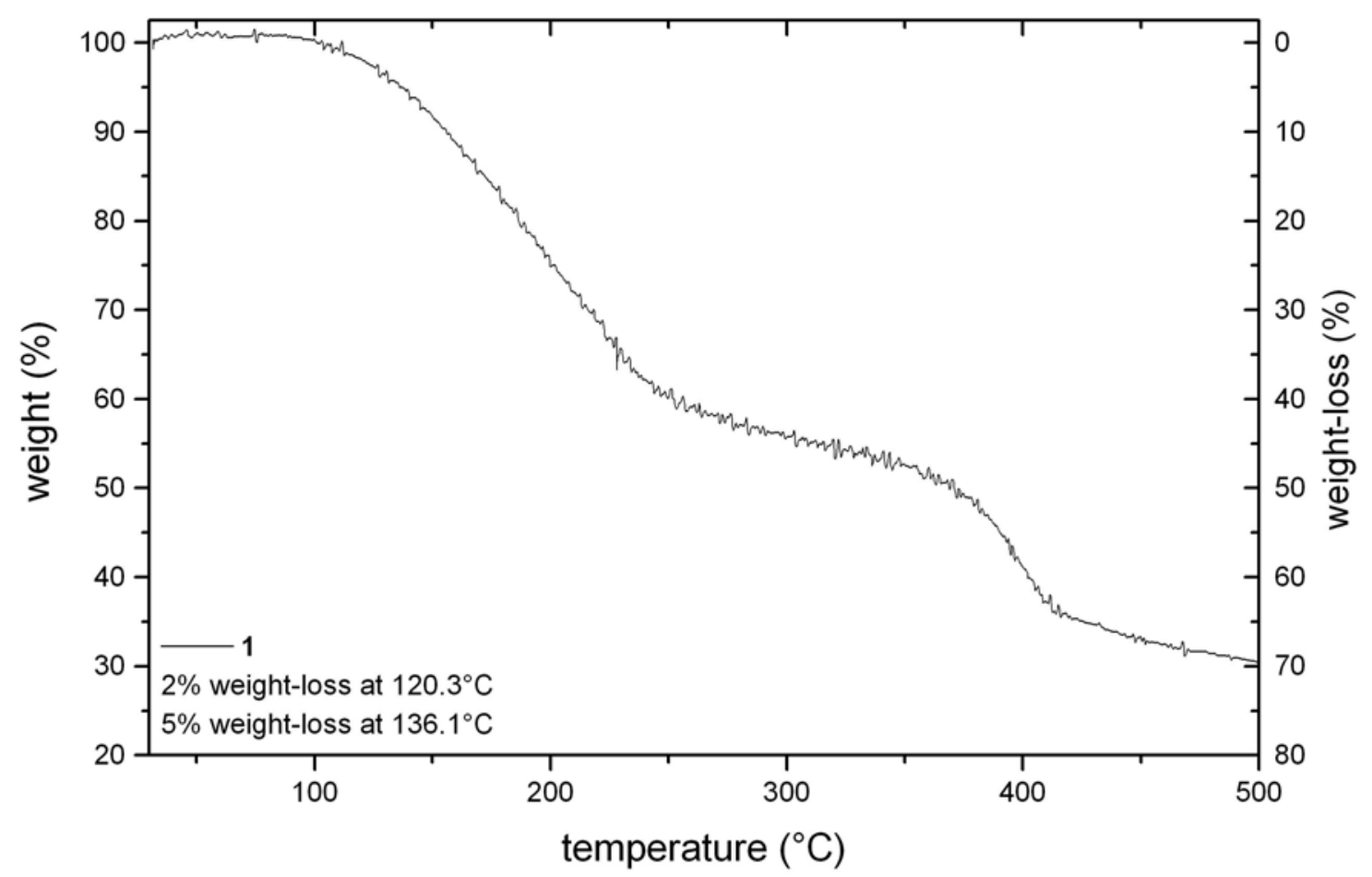

Figure S3. TGA run of 1

Preparation of (OC-6-42)-bis $\left(\kappa^{2}\left(C^{2}, N\right)\right.$-2-phenylpyridine $)\left(\kappa^{2}(N, O)\right.$-5-formyl-8-quinolinolate) iridium(III) (2)



Di- $\mu$-chlorotetrakis $\left(\kappa^{2}\left(C^{2}, N\right)\right.$-2-phenylpyridine $)$ diiridium(III) $(0.0500 \mathrm{~g}, 0.047 \mathrm{mmol}, 1$ eq. $)$ and 8-hydroxyquinoline-5-carbaldehyde $(0.0184 \mathrm{~g}, 0.106 \mathrm{mmol}, 2.3 \text { eq. })^{\mathrm{S} 3}$ were suspended in a solvent-mixture of degassed $\mathrm{CH}_{2} \mathrm{Cl}_{2} / \mathrm{EtOH} / \mathrm{Et}_{3} \mathrm{~N}(2000 \mu \mathrm{L} / 1000 \mu \mathrm{L} / 300 \mu \mathrm{L})$ under inert atmosphere of argon. The suspension was refluxed at $80^{\circ} \mathrm{C}$ for 20 hours by which the mixture turned orange.

The solvent was removed in vacuo and the residue was washed with methanol. Filtering off the orange precipitate gave the product in pure form. Yield: $47.4 \mathrm{mg}(75.5 \%)$. $\mathrm{R}_{\mathrm{f}}($ acetone $)=0.88$.

${ }^{1} \mathrm{H}-\mathrm{NMR}\left(\delta, 20^{\circ} \mathrm{C}, \mathrm{DMSO}_{\mathrm{d}}, 500 \mathrm{MHz}\right): 9.90(\mathrm{~s}, 1 \mathrm{H}, \mathrm{CHO}), 9.54\left(\mathrm{dd}, 1 \mathrm{H},{ }^{3} \mathrm{~J}_{\mathrm{HH}}=8.5 \mathrm{~Hz}\right.$, $\left.{ }^{4} J_{H H}=1.5 \mathrm{~Hz}, \mathrm{q}^{4}\right), 9.39\left(\mathrm{~d}, 1 \mathrm{H},{ }^{3} J_{H H}=5.9 \mathrm{~Hz}, \mathrm{py}^{6}\right), 8.20\left(\mathrm{~d}, 1 \mathrm{H},{ }^{3} J_{H H}=8.3 \mathrm{~Hz}, \mathrm{py}^{3}\right), 8.18(\mathrm{~d}$,

${ }^{\text {s3 }}$ Clemo, G. R.; Howe, R. J. J. Chem. Soc. 1955, 3552.

ESI 6 
$\left.1 \mathrm{H},{ }^{3} J_{\mathrm{HH}}=8.3 \mathrm{~Hz}, \mathrm{py}^{3}\right), 7.98\left(\mathrm{~d}, 1 \mathrm{H},{ }^{3} J_{\mathrm{HH}}=8.5 \mathrm{~Hz}, \mathrm{q}^{6}\right), 7.90-7.80\left(\mathrm{~m}, 4 \mathrm{H}, \mathrm{py}^{4}, \mathrm{ph}^{6}\right), 7.71$ $\left(\mathrm{dd}, 1 \mathrm{H},{ }^{3} J_{H H}=4.9 \mathrm{~Hz},{ }^{4} J_{H H}=1.5 \mathrm{~Hz}, \mathrm{q}^{2}\right), 7.64-7.62\left(\mathrm{~m}, 1 \mathrm{H}, \mathrm{q}^{3}\right), 7.51\left(\mathrm{~d}, 1 \mathrm{H},{ }^{3} J_{H H}=5.6 \mathrm{~Hz}\right.$, py $\left.^{6}\right), 7.28-7.25\left(\mathrm{ddd}, 1 \mathrm{H}, 2 \mathrm{x}^{3} J_{\mathrm{HH}}=\right.$ ca. $7.0 \mathrm{~Hz},{ }^{4} J_{\mathrm{HH}}=1.2 \mathrm{~Hz}$, py $\left.{ }^{5}\right), 7.11-7.08(\mathrm{ddd}, 1 \mathrm{H}, 2 \mathrm{x}$ ${ }^{3} J_{H H}=$ ca. $\left.7.0 \mathrm{~Hz},{ }^{4} J_{H H}=1.2 \mathrm{~Hz}, \mathrm{py}^{5}\right), 6.92\left(\mathrm{ddd}, 1 \mathrm{H}, 2 \mathrm{x}^{3} J_{\mathrm{HH}}=\right.$ ca. $\left.7.3 \mathrm{~Hz},{ }^{4} J_{H H}=1.0 \mathrm{~Hz}, \mathrm{ph}^{5}\right)$, $6.88\left(\mathrm{ddd}, 1 \mathrm{H}, 2 \mathrm{x}{ }^{3} J_{\mathrm{HH}}=\right.$ ca. $\left.7.3 \mathrm{~Hz},{ }^{4} J_{H H}=1.0 \mathrm{~Hz}, \mathrm{ph}^{5}\right), 6.82-6.78,6.73(\mathrm{~m}, 2 \mathrm{H}$, ddd, $1 \mathrm{H}$, $2 \mathrm{x}^{3} J_{H H}=$ ca. $\left.7.3 \mathrm{~Hz},{ }^{4} J_{H H}=1.2 \mathrm{~Hz}, \mathrm{ph}^{4}, \mathrm{q}^{7}\right), 6.29\left(\mathrm{~d}, 1 \mathrm{H},{ }^{3} J_{H H}=7.6 \mathrm{~Hz}, \mathrm{ph}^{3}\right)$ and $6.12(\mathrm{~d}, 1 \mathrm{H}$, $\left.{ }^{3} J_{H H}=7.6 \mathrm{~Hz}, \mathrm{ph}^{3}\right)$.

${ }^{13} \mathrm{C}-\mathrm{NMR}\left(\delta, 20^{\circ} \mathrm{C}, \mathrm{DMSO}_{\mathrm{d}}, 125 \mathrm{MHz}\right): 189.5(1 \mathrm{C}, \mathrm{CHO}), 176.0\left(1 \mathrm{C}, \mathrm{q}^{8}\right), 167.8,167.0(2 \mathrm{C}$, $\left.\mathrm{py}^{2}\right), 150.5,148.4\left(2 \mathrm{C}, \mathrm{ph}^{2}\right), 148.2,147.7,146.8\left(3 \mathrm{C}, \mathrm{py}^{6}, \mathrm{q}^{2}\right), 144.7,144.2,143.2\left(3 \mathrm{C}, \mathrm{ph}^{1}\right.$, $\left.\mathrm{q}^{8 \mathrm{a}}\right), 142.5\left(1 \mathrm{C}, \mathrm{q}^{6}\right), 138.1,138.0,134.5,132.0,131.7,129.6,129.0,125.8,124.7,124.3$, 123.3, 123.0, 121.2, 120.9, 119.4, $119.3\left(16 \mathrm{C}, \mathrm{ph}^{3,4,5,6}, \mathrm{py}^{3,4,5}, \mathrm{q}^{3,4}\right), 130.3\left(1 \mathrm{C}, \mathrm{q}^{4 \mathrm{a}}\right), 116.0(1 \mathrm{C}$, $\left.\mathrm{q}^{5}\right)$ and $115.8\left(1 \mathrm{C}, \mathrm{q}^{7}\right)$.

MALDI MS (m/z): [ $\left.\mathrm{C}_{32} \mathrm{H}_{22} \mathrm{IrN}_{3} \mathrm{O}_{2}\right] 671.1387$ (calcd 671.1318).

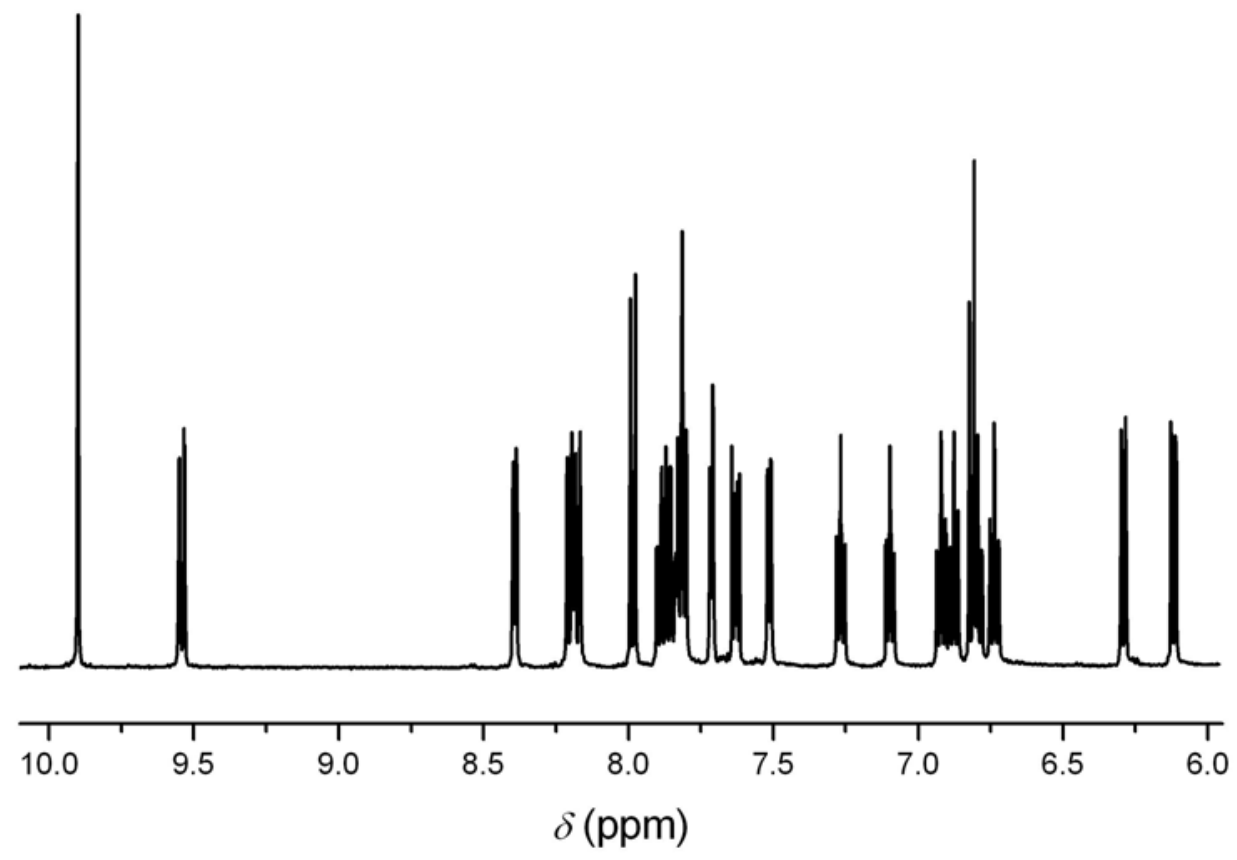

Figure S4. ${ }^{1} \mathrm{H}-\mathrm{NMR}$ spectrum of 2 in $\mathrm{DMSO}-\mathrm{d}_{6}$ 


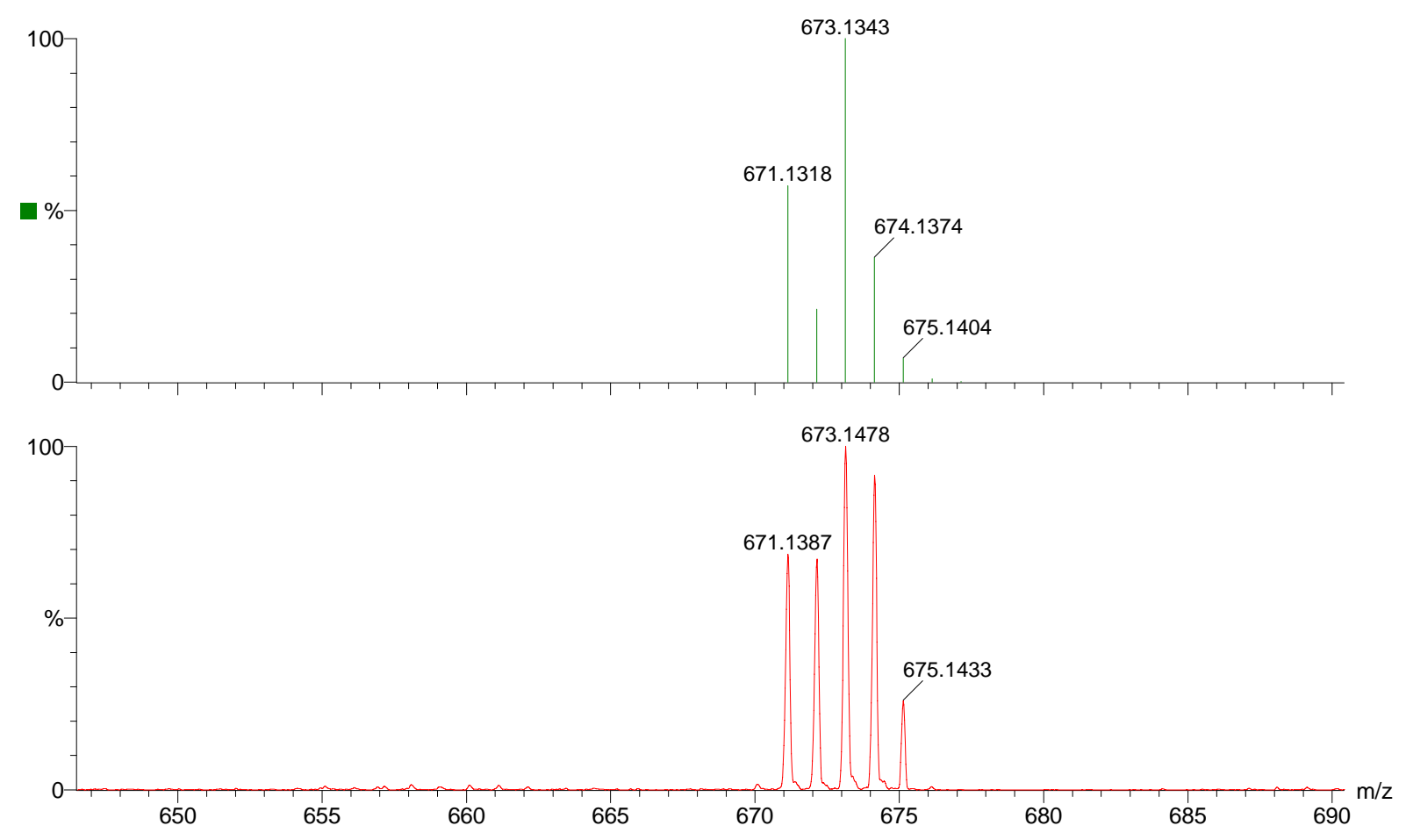

Figure S5. Calculated (above) and measured (below) isotope pattern of 2



Figure S6. TGA run of 2 
Preparation of (OC-6-42)-bis $\left(\kappa^{2}\left(C^{2}, N\right)\right.$-2-phenylpyridine $)\left(\kappa^{2}(N, O)-5\right.$-nitro-8-quinolinolate) iridium(III) (3)
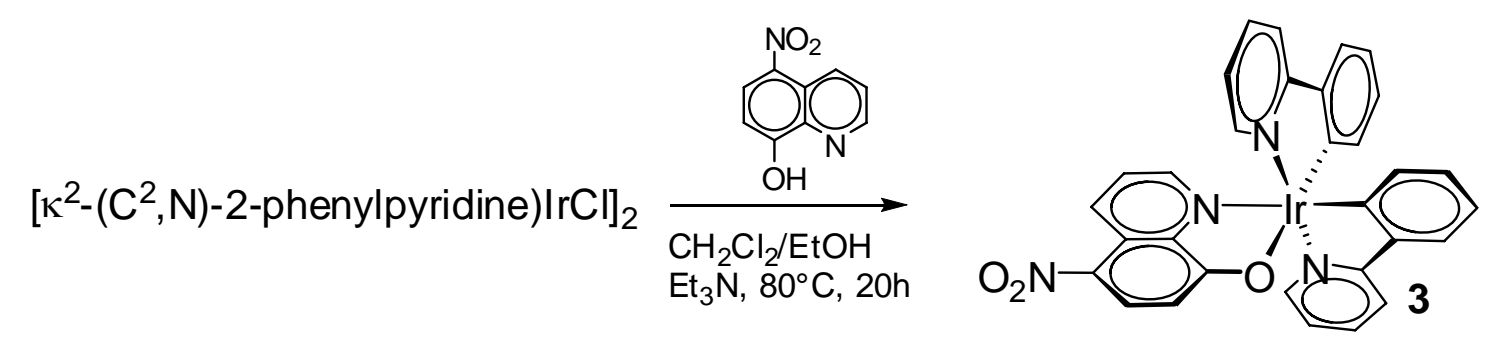

Di- $\mu$-chlorotetrakis $\left(\kappa^{2}\left(C^{2}, N\right)\right.$-2-phenylpyridine)diiridium(III) $(0.1010 \mathrm{~g}, 0.094 \mathrm{mmol}, 1$ eq. $)$ and 5-nitro-8-hydroxyquinoline $(0.0446 \mathrm{~g}, 0.235 \mathrm{mmol}, 2.5 \mathrm{eq}$.) were suspended in a solventmixture of degassed $\mathrm{CH}_{2} \mathrm{Cl}_{2} / \mathrm{EtOH} / \mathrm{Et}_{3} \mathrm{~N}(4000 \mu \mathrm{L} / 2000 \mu \mathrm{L} / 600 \mu \mathrm{L})$ under inert atmosphere of argon. The suspension was refluxed at $80^{\circ} \mathrm{C}$ for 20 hours by which the mixture turned red. The solvent was removed in vacuo and the residue was washed with methanol. Filtering off the red precipitate gave the product in pure form. Yield: $109.1 \mathrm{mg}(84.0 \%)$. $\mathrm{R}_{\mathrm{f}}(\mathrm{cy}: \mathrm{ee}=1: 1)=0.57$.

${ }^{1} \mathrm{H}-\mathrm{NMR}\left(\delta, 20^{\circ} \mathrm{C}\right.$, DMSO-d $\left.6,500 \mathrm{MHz}\right): 9.32-9.30\left(\mathrm{~m}, 1 \mathrm{H}, \mathrm{q}^{4}\right), 8.58\left(\mathrm{dd}, 1 \mathrm{H},{ }^{3} \mathrm{~J}_{\mathrm{HH}}=9.5\right.$ $\left.\mathrm{Hz},{ }^{4} J_{H H}=1.4 \mathrm{~Hz}, \mathrm{q}^{6}\right), 8.33\left(\mathrm{~d}, 1 \mathrm{H},{ }^{3} J_{H H}=5.6 \mathrm{~Hz}, \mathrm{py}^{6}\right), 8.21-8.19\left(\mathrm{dd}, 2 \mathrm{H}, 2 \mathrm{x}^{3} J_{H H}=\right.$ ca. 8.6 $\left.\mathrm{Hz}, \mathrm{py}^{3}\right), 7.92-7.81\left(\mathrm{~m}, 4 \mathrm{H}, \mathrm{py}^{4}, \mathrm{ph}^{6}\right), 7.74\left(\mathrm{~m}, 2 \mathrm{H}, \mathrm{q}^{2,3}\right), 7.55\left(\mathrm{~d}, 1 \mathrm{H},{ }^{3} J_{\mathrm{HH}}=5.9 \mathrm{~Hz}, \mathrm{py}^{6}\right)$, $7.29-7.26\left(\mathrm{ddd}, 1 \mathrm{H}, 2 \mathrm{x}{ }^{3} J_{H H}=\right.$ ca. $7.0 \mathrm{~Hz},{ }^{4} J_{H H}=$ ca. $\left.1.2 \mathrm{~Hz}, \mathrm{py}{ }^{5}\right), 7.12-7.10(\mathrm{ddd}, 1 \mathrm{H}, 2 \mathrm{x}$ ${ }^{3} J_{H H}=$ ca. $7.1 \mathrm{~Hz},{ }^{4} J_{H H}=$ ca. $\left.1.2 \mathrm{~Hz}, \mathrm{py}^{5}\right), 6.95-6.92\left(\mathrm{ddd}, 1 \mathrm{H}, 2 \mathrm{x}{ }^{3} J_{\mathrm{HH}}=\right.$ ca. $7.4 \mathrm{~Hz},{ }^{4} J_{\mathrm{HH}}=$ ca. $\left.1.0 \mathrm{~Hz}, \mathrm{ph}^{5}\right), 6.90-6.87\left(\mathrm{ddd}, 1 \mathrm{H}, 2 \mathrm{x}^{3} J_{\mathrm{HH}}=\right.$ ca. $7.3 \mathrm{~Hz},{ }^{4} J_{\mathrm{HH}}=$ ca. $\left.1.0 \mathrm{~Hz}, \mathrm{ph}^{5}\right), 6.82-$ $6.77\left(\mathrm{~m}, 2 \mathrm{H}, \mathrm{q}^{7}, \mathrm{ph}^{4}\right), 6.76-6.73\left(\mathrm{ddd}, 1 \mathrm{H}, 2 \mathrm{x}^{3} J_{\mathrm{HH}}=\right.$ ca. $7.4 \mathrm{~Hz},{ }^{4} J_{\mathrm{HH}}=$ ca. $\left.1.2 \mathrm{~Hz}, \mathrm{ph}^{4}\right), 6.27$ $\left(\mathrm{d}, 1 \mathrm{H},{ }^{3} J_{\mathrm{HH}}=7.8 \mathrm{~Hz}, \mathrm{ph}^{3}\right)$ and $6.10\left(\mathrm{~d}, 1 \mathrm{H},{ }^{3} J_{\mathrm{HH}}=7.8 \mathrm{~Hz}, \mathrm{ph}^{3}\right)$.

${ }^{13} \mathrm{C}-\mathrm{NMR}\left(\delta, 20^{\circ} \mathrm{C}\right.$, DMSO-d $\left.6,125 \mathrm{MHz}\right): 177.8\left(1 \mathrm{C}, \mathrm{q}^{8}\right), 167.7,166.9\left(2 \mathrm{C}, \mathrm{py}^{2}\right), 149.8$, $147.7\left(2 \mathrm{C}, \mathrm{ph}^{2}\right), 148.4,147.8,147.0\left(3 \mathrm{C}, \mathrm{py}^{6}, \mathrm{q}^{2}\right), 144.6,144.1,142.1\left(3 \mathrm{C}, \mathrm{ph}^{1}, \mathrm{q}^{8 \mathrm{a}}\right), 138.3$, 138.2, 134.1, 132.4, 132.0, 131.6, 129.7, 129.1, 126.9 (evtl. 2C), 124.8, 124.3, 123.4, 123.1, $121.4,121.1,119.5\left(17 \mathrm{C}, \mathrm{q}^{3,4,6}, \mathrm{ph}^{3,4,5,6}, \mathrm{py}^{3,4,5}\right), 128.1\left(1 \mathrm{C}, \mathrm{q}^{4 \mathrm{a}}\right), 114.8\left(1 \mathrm{C}, \mathrm{q}^{7}\right)$ and $109.3\left(1 \mathrm{C}, \mathrm{q}^{5}\right)$.

MALDI MS (m/z): [ $\left.\mathrm{C}_{31} \mathrm{H}_{21} \mathrm{IrN}_{4} \mathrm{O}_{3}\right] 688.1229$ (calcd 688.1219). 


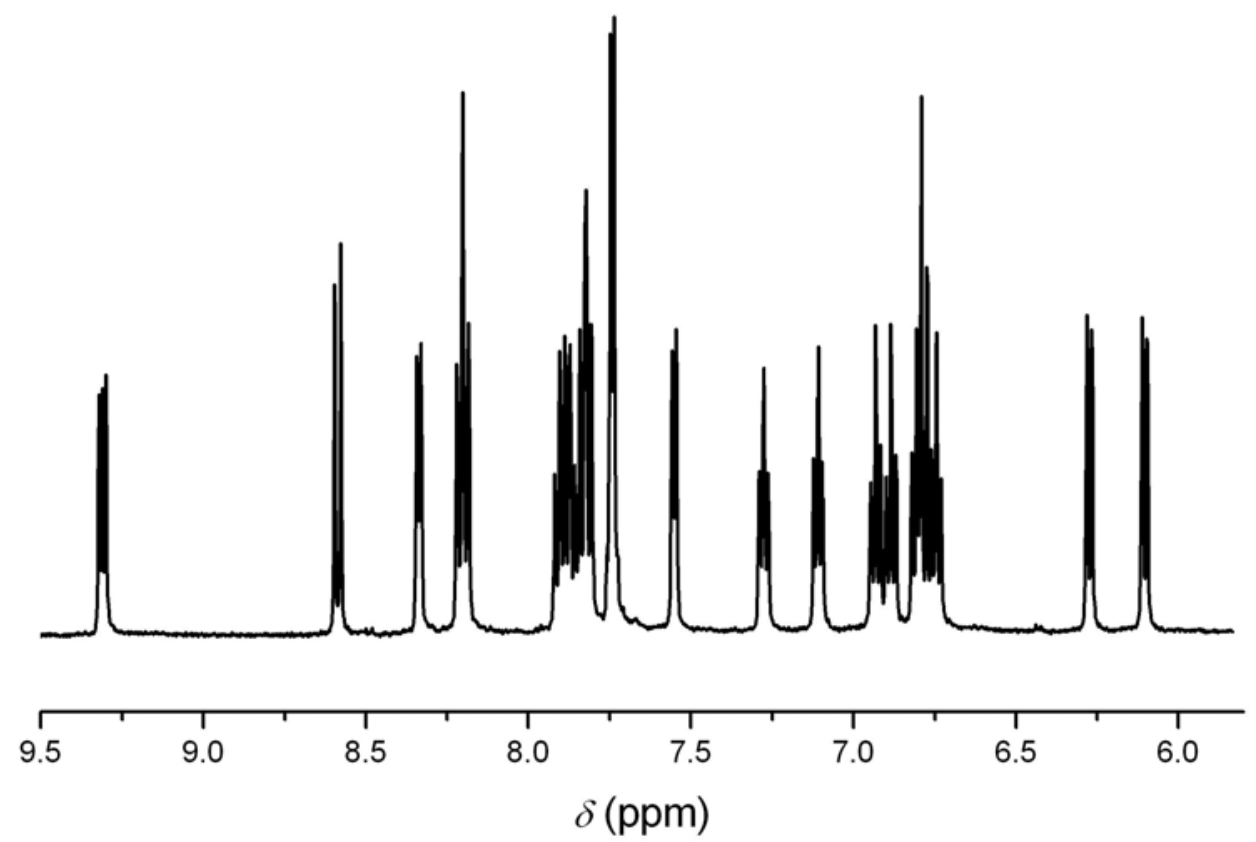

Figure S7. ${ }^{1} \mathrm{H}-\mathrm{NMR}$ spectrum of 3 in $\mathrm{DMSO}-\mathrm{d}_{6}$

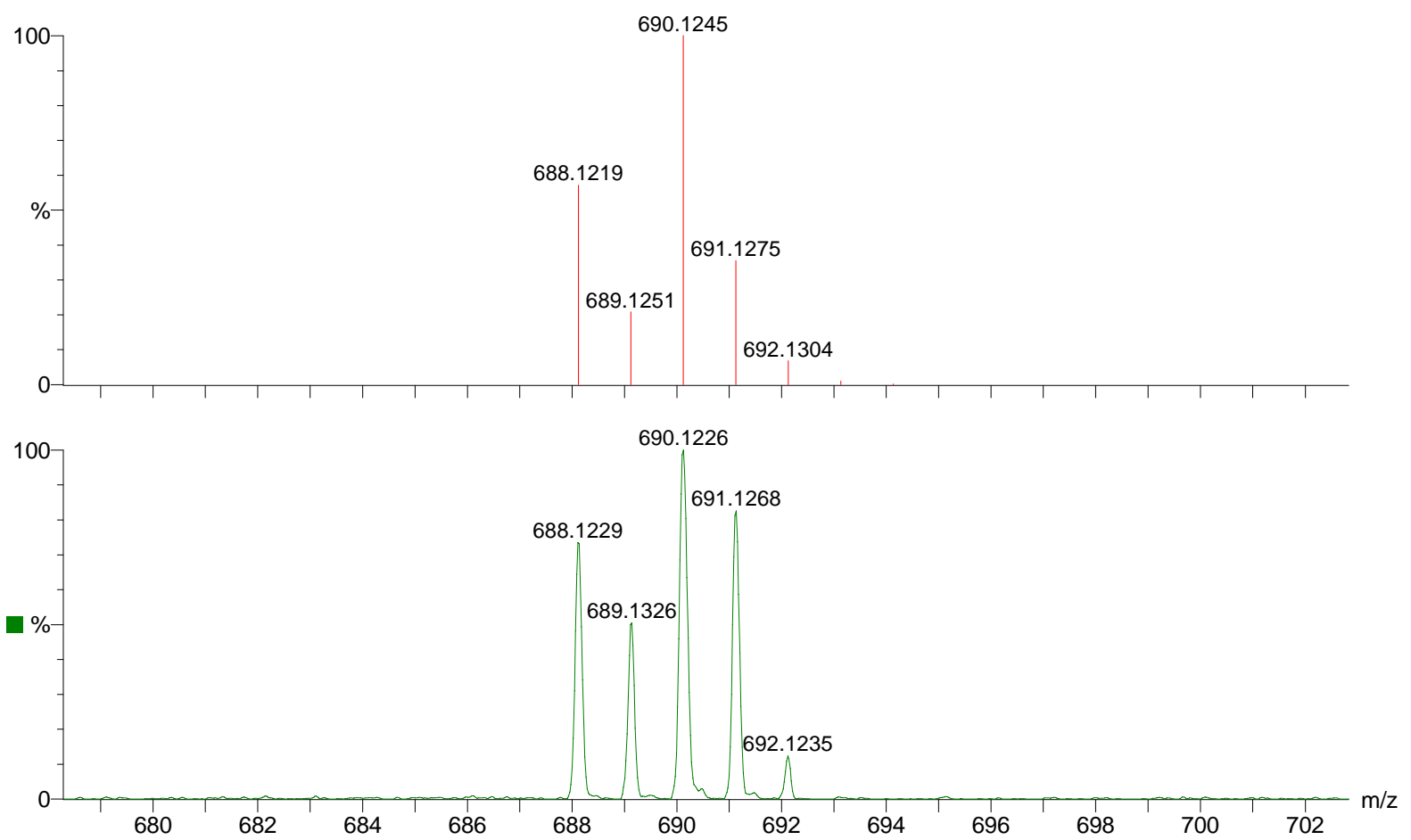

Figure S8. Calculated (above) and measured (below) isotope pattern of $\mathbf{3}$ 


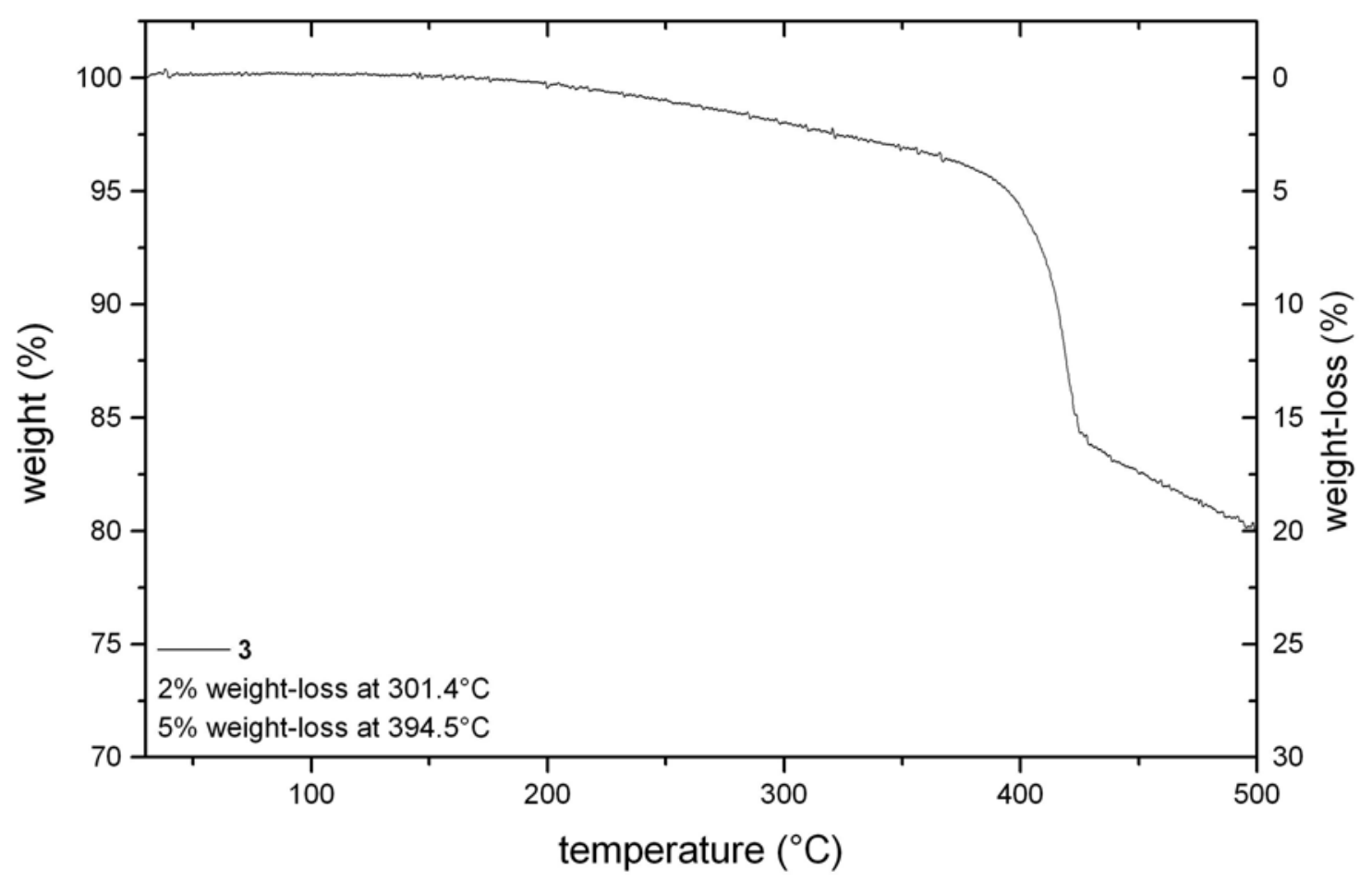

Figure S9. TGA run of 3

Single crystals of 3 suitable for X-ray diffraction were obtained from ether diffusion into saturated $\mathrm{CH}_{2} \mathrm{Cl}_{2}$ solutions. X-ray data are summarized subsequently, graphics are shown in Fig. S10.


Figure S10. OC-6-42 stereochemistry (left) and packing diagram of 3 (right) 
Crystal data and structure refinement for 3

\begin{tabular}{|c|c|}
\hline Identification code & $1158 \mathrm{fr}$ \\
\hline Empirical formula & C31 H21 Ir N4 O3 \\
\hline Formula weight & 689.72 \\
\hline Temperature & $173(2) \mathrm{K}$ \\
\hline Wavelength & $0.71073 \AA$ \\
\hline Crystal system, space group & Monoclinic, $\mathrm{P} 2(1) / \mathrm{n}$ \\
\hline Unit cell dimensions & $\begin{array}{l}\mathrm{a}=8.0795(5) \AA \quad \alpha=90 \mathrm{deg} . \\
\mathrm{b}=23.4650(14) \AA \quad \beta=93.062(1) \mathrm{deg} . \\
\mathrm{c}=13.1145(8) \AA \quad \gamma=90 \mathrm{deg} .\end{array}$ \\
\hline Volume & $2482.8(3) \AA^{3}$ \\
\hline Z, Calculated density & $4,1.845 \mathrm{Mg} / \mathrm{m}^{3}$ \\
\hline Absorption coefficient & $5.421 \mathrm{~mm}^{-1}$ \\
\hline $\mathrm{F}(000)$ & 1344 \\
\hline Crystal size & $0.24 \times 0.15 \times 0.14 \mathrm{~mm}$ \\
\hline Diffractometer & $\begin{array}{l}\text { Bruker Smart APEX CCD 3-circle (sealed X-ray } \\
\text { tube, Mo K } \alpha \text { rad., graphite monochromator } \\
\text { detector.distance } 50 \mathrm{~mm}, 512 \times 512 \text { pixels) }\end{array}$ \\
\hline Scan type / width / speed frames & $\begin{array}{l}\Delta \omega=0.3 \mathrm{deg} / 10 \mathrm{sec} \text { per frame } \\
\text { full sphere data collection, } 4 \times 600 \text { frames }\end{array}$ \\
\hline Theta range for data collection & 2.67 to $30.00 \mathrm{deg}$. \\
\hline Index ranges & $-11<=\mathrm{h}<=11,-33<=\mathrm{k}<=33,-18<=1<=18$ \\
\hline Reflections collected / unique & $36268 / 7232[\mathrm{R}(\mathrm{int})=0.0270]$ \\
\hline Completeness to $\theta=30.00$ & $99.8 \%$ \\
\hline Absorption correction & Multi-scan (program SADABS; G. M. Sheldrick, 1996) \\
\hline Max. and min. transmission & 0.47 and 0.30 \\
\hline Structure solution & Direct methods (program SHELXS97) \\
\hline Refinement method & Full-matrix least-squares on $\mathrm{F}^{2}$ (prg SHELXL97) \\
\hline Data / restraints / parameters & $7232 / 0 / 352$ \\
\hline Goodness-of-fit on $\mathrm{F}^{2}$ & 1.052 \\
\hline Final $R$ indices $[I>2 \sigma(I)]$ & $\mathrm{R} 1=0.0485, \mathrm{wR} 2=0.1022$ \\
\hline $\mathrm{R}$ indices (all data) & $\mathrm{R} 1=0.0591, \mathrm{wR} 2=0.1083$ \\
\hline Largest diff. peak and hole & 2.71 and $-2.113 \mathrm{e} \AA^{-3}$ \\
\hline
\end{tabular}

$\mathrm{R} 1=\Sigma|| \mathrm{F}_{\mathrm{o}}|-| \mathrm{F}_{\mathrm{c}}|| / \Sigma\left|\mathrm{F}_{\mathrm{o}}\right|, \mathrm{wR} 2=\left[\Sigma\left(\mathrm{w}\left(\mathrm{F}_{\mathrm{o}}^{2}-\mathrm{F}_{\mathrm{c}}{ }^{2}\right)^{2}\right) / \Sigma\left(\mathrm{w}\left(\mathrm{F}_{\mathrm{o}}{ }^{2}\right)^{2}\right)\right]^{1 / 2}$

\section{Lifetime Measurements and Thin Film Spectra}

Sample Preparation. Polymer cocktails were prepared by dissolving $2 \mathrm{mg}$ of the corresponding complex and $100 \mathrm{mg}$ of polystyrene in $1 \mathrm{~mL} \mathrm{CHCl}$. The polymer solutions were suck into a Pasteur pipette and the excess amount of the solution was blown out with compressed air. The resulting films were dried in a drying chamber at $70^{\circ} \mathrm{C}$ for $12 \mathrm{~h}$. 
Lifetimes at different Modulation Frequencies.

Table S1. Lifetime measurements

\begin{tabular}{ccccc}
\hline \multirow{2}{*}{ Compound } & Conditions & $\begin{array}{c}\text { Lifetime at } \\
5 \mathrm{kHz} / \mu \mathrm{s}^{a}\end{array}$ & $\begin{array}{c}\text { Lifetime at } \\
10 \mathrm{kHz} / \mu \mathrm{s}^{a}\end{array}$ & $\begin{array}{c}\text { Lifetime at } \\
15 \mathrm{kHz} / \mu \mathrm{s}^{a}\end{array}$ \\
\hline \multirow{2}{*}{$\mathbf{2}$} & air & 11 & 13 & 14 \\
& nitrogen & 24 & 25 & 28 \\
\hline \multirow{2}{*}{3} & air & 8 & 7 & 7 \\
& nitrogen & 16 & 13 & 11 \\
\hline
\end{tabular}

${ }^{a}$ Estimated experimental error: $\pm 2 \mu \mathrm{s}$

Thin Film Spectra of Coated Capillaries.

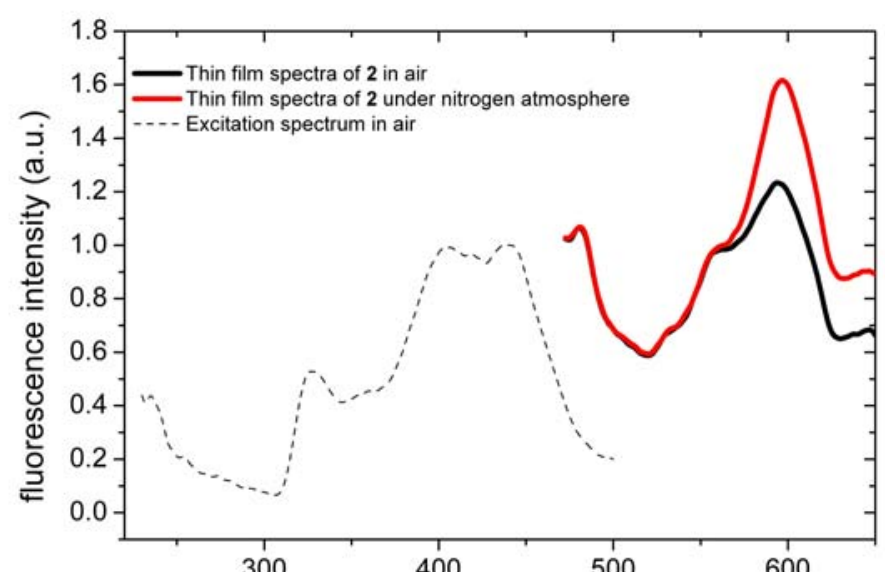

Figure S11. Thin film spectra of coated capillaries of $2\left(\lambda_{\mathrm{exc}}=400 \mathrm{~nm}\right)$

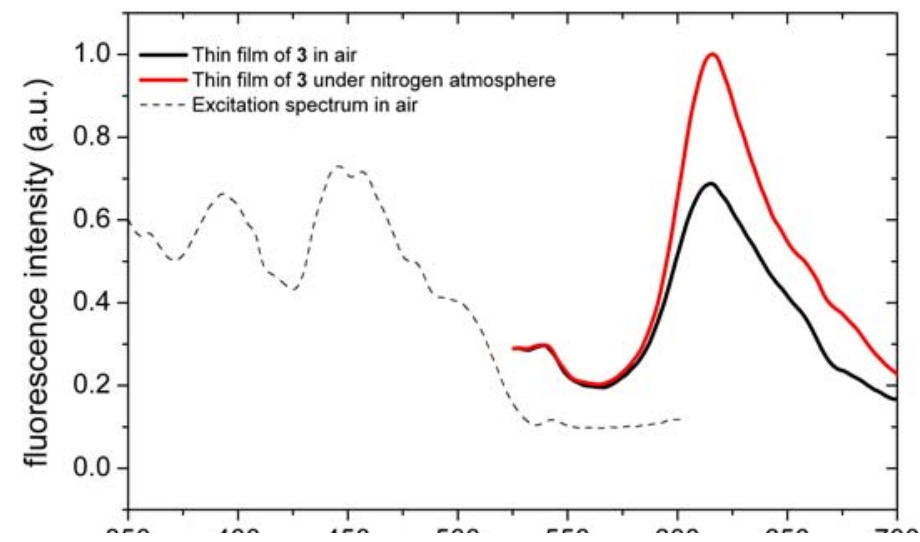

Figure S12. Thin film spectra of coated capillaries of $3\left(\lambda_{\mathrm{exc}}=450 \mathrm{~nm}\right)$ 


\section{Electrochemical Measurements}

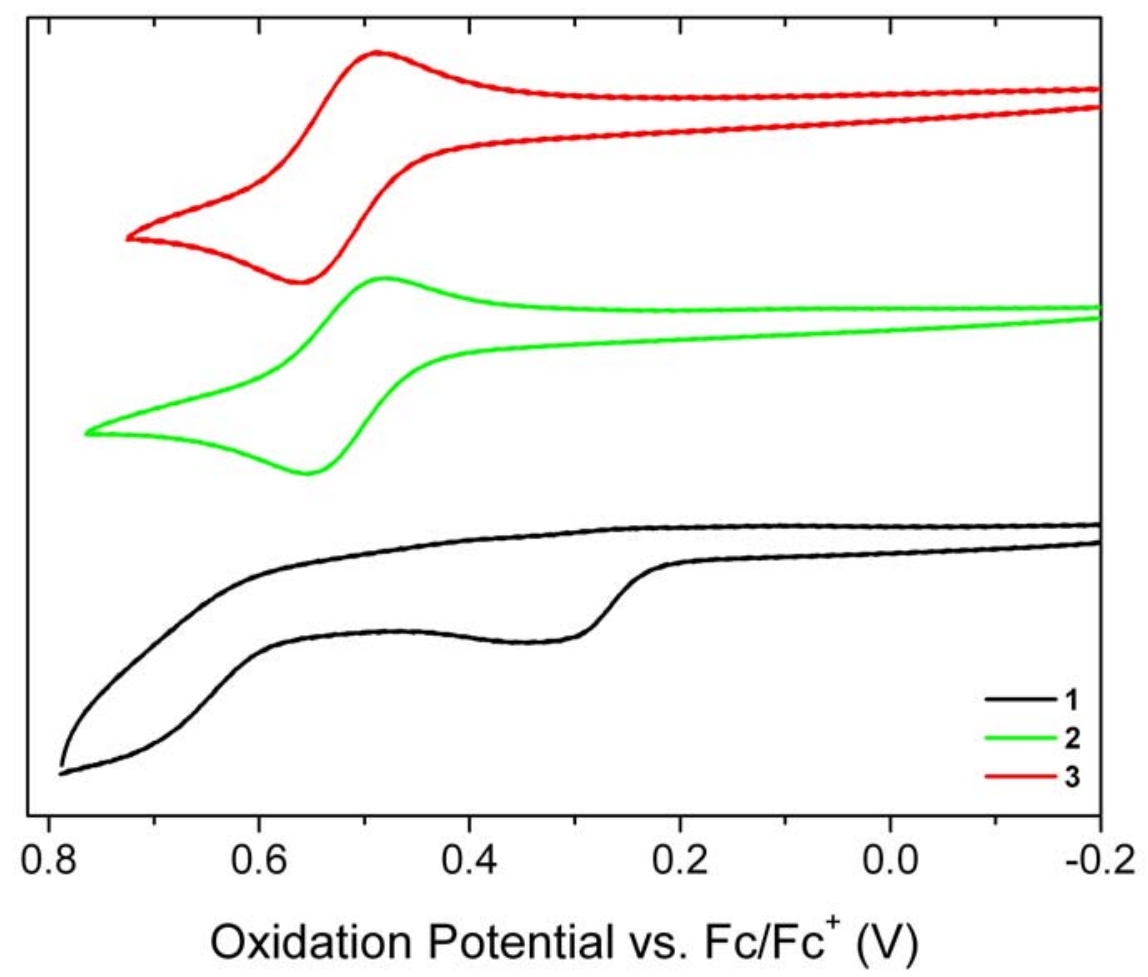

Figure S13. Cyclic voltammograms of compounds $\mathbf{1}-\mathbf{3}$ (anodic runs, performed in $\mathrm{CH}_{2} \mathrm{Cl}_{2} / \mathrm{Bu}_{4} \mathrm{NPF}_{6}$ as electrolyte, substrate concentration approx. $1 \mathrm{mmol} / \mathrm{L}$, scan rate was $0.1 \mathrm{~V} / \mathrm{s}$, currents are normalized to the concentration)

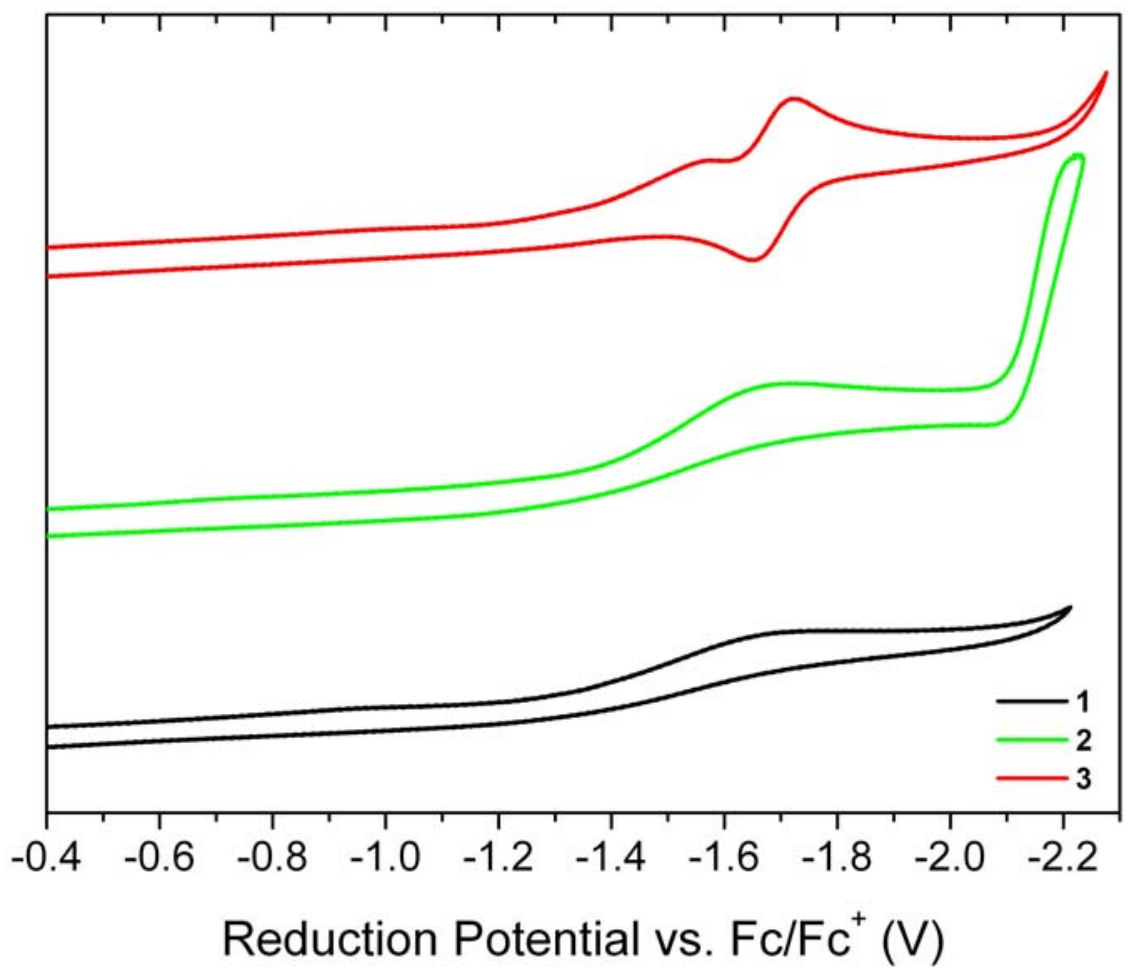

Figure S14. Cyclic voltammograms of compounds $1-3$ (cathodic runs, performed in $\mathrm{CH}_{2} \mathrm{Cl}_{2} / \mathrm{Bu}_{4} \mathrm{NPF}_{6}$ as electrolyte, substrate concentration approx. $1 \mathrm{mmol} / \mathrm{L}$, scan rate was $0.1 \mathrm{~V} / \mathrm{s}$, currents are normalized to the concentration) 
Cyclic voltammetry (CV) was applied to investigate the electrochemical behavior and to estimate the energy levels of the highest occupied molecular orbital (HOMO) and the lowest unoccupied molecular orbital (LUMO). While for $\mathbf{1}$ an irreversible oxidation peak at $0.32 \mathrm{~V}$ (peak potential) vs. ferrocene/ferrocenium is observed, $\mathbf{2}$ and $\mathbf{3}$ show a chemically reversible peak at higher oxidation potentials giving thermo dynamical redox potentials of $0.518 \mathrm{~V}$ and $0.524 \mathrm{~V}$, respectively. Peak separation values between 69 and $72 \mathrm{mV}$ indicate quasi-reversible electron transfer processes. Reduction experiments exhibit a broad wave starting at $-1.2 \mathrm{~V}$ resulting a peak potential at $-1.7 \mathrm{~V}$ for $\mathbf{1}$. Similar reduction behavior is obtained for 2 with an additional follow-up reduction at $-2.226 \mathrm{~V} .3$ shows the corresponding irreversible reduction wave at $-1.6 \mathrm{~V}$ followed by an overlapping chemically reversible redox peak at $-1.688 \mathrm{~V}$ and a peak separation of $71 \mathrm{mV}$.

Table S2. Results of electrochemical measurements

\begin{tabular}{ccccc}
\hline \multirow{2}{*}{ Compound } & $\begin{array}{c}\text { Oxidation Onset } \\
\text { / V }\end{array}$ & $I_{\mathrm{P}} / \mathrm{eV}$ & $\begin{array}{c}\text { Reduction Onset } \\
/ \mathrm{V}\end{array}$ & \multirow{2}{*}{$E_{\mathrm{A}} / \mathrm{eV}$} \\
\hline $\mathbf{1}$ & 0.22 & -5.0 & -1.3 & -3.5 \\
$\mathbf{2}$ & 0.42 & -5.2 & -1.3 & -3.5 \\
$\mathbf{3}$ & 0.43 & -5.3 & -1.3 & -3.5 \\
\hline
\end{tabular}

\section{Organic Light-Emitting Devices (OLEDs)}

\section{Host Materials.}

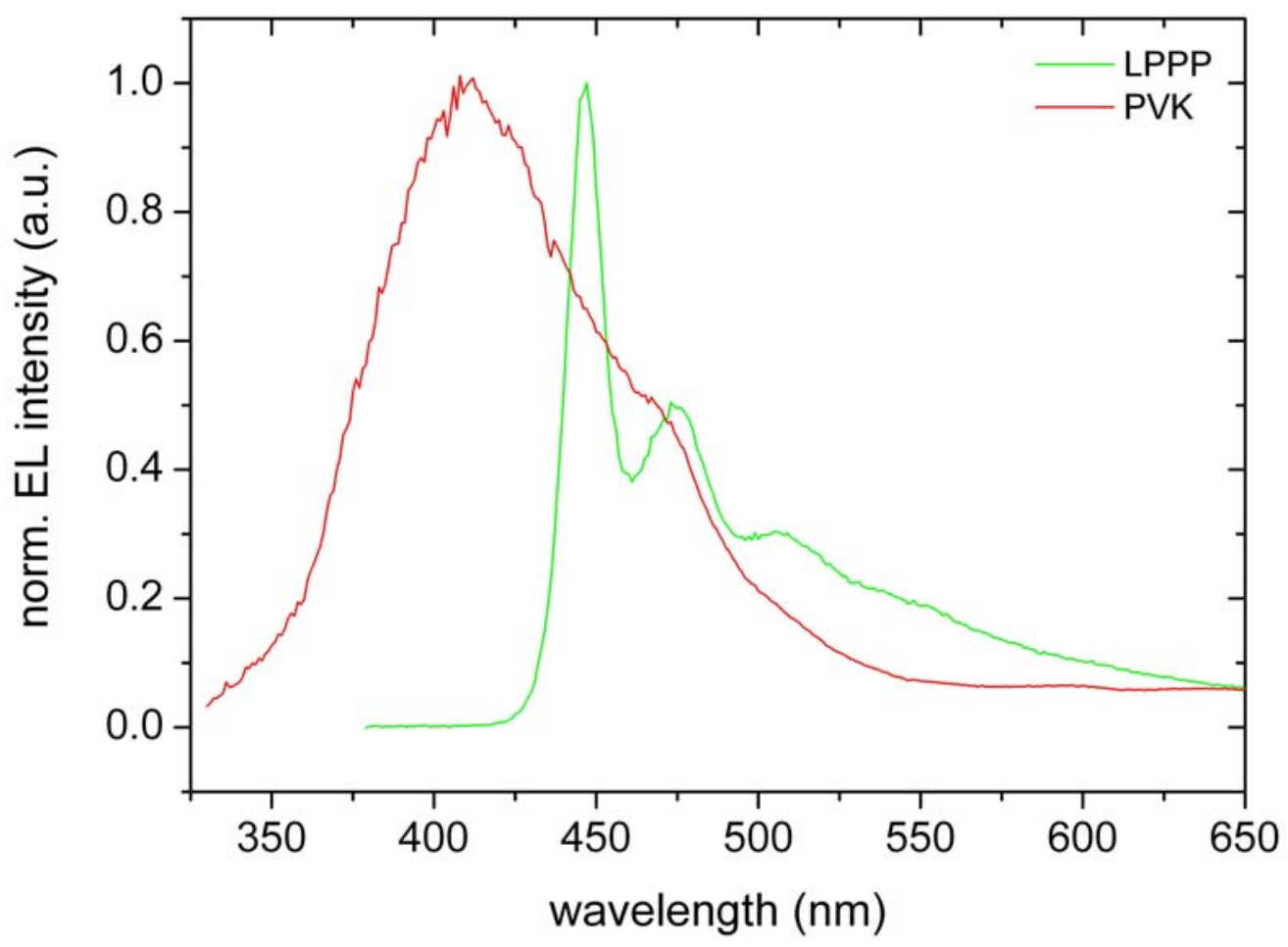

Figure S15. Normalized electroluminescence spectra of PVK and LPPP 




Figure S16. Normalized electroluminescence spectrum of 3 in PVK

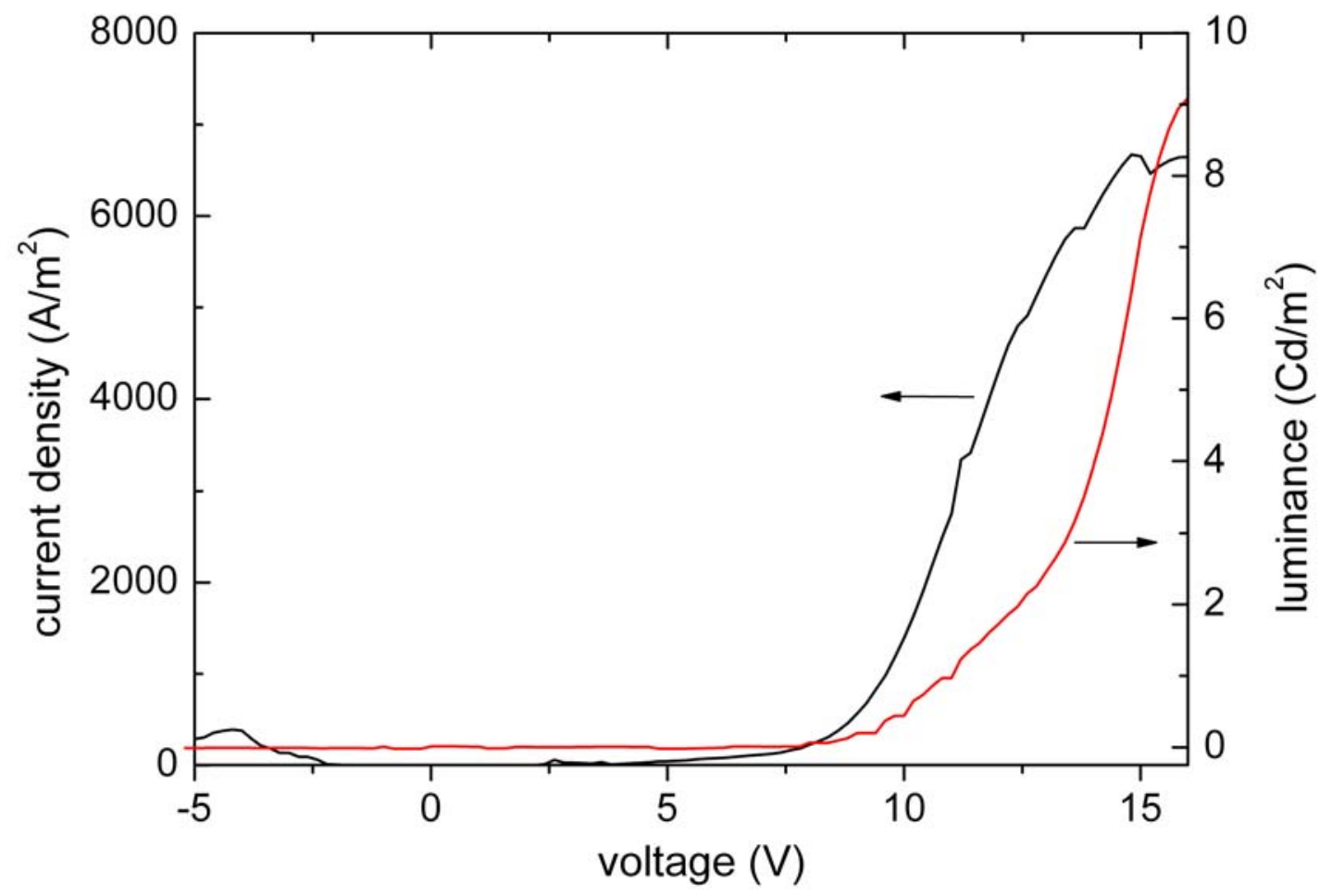

Figure S17. Bias/current characteristics and bias/electroluminescence characteristics of 3 in PVK 


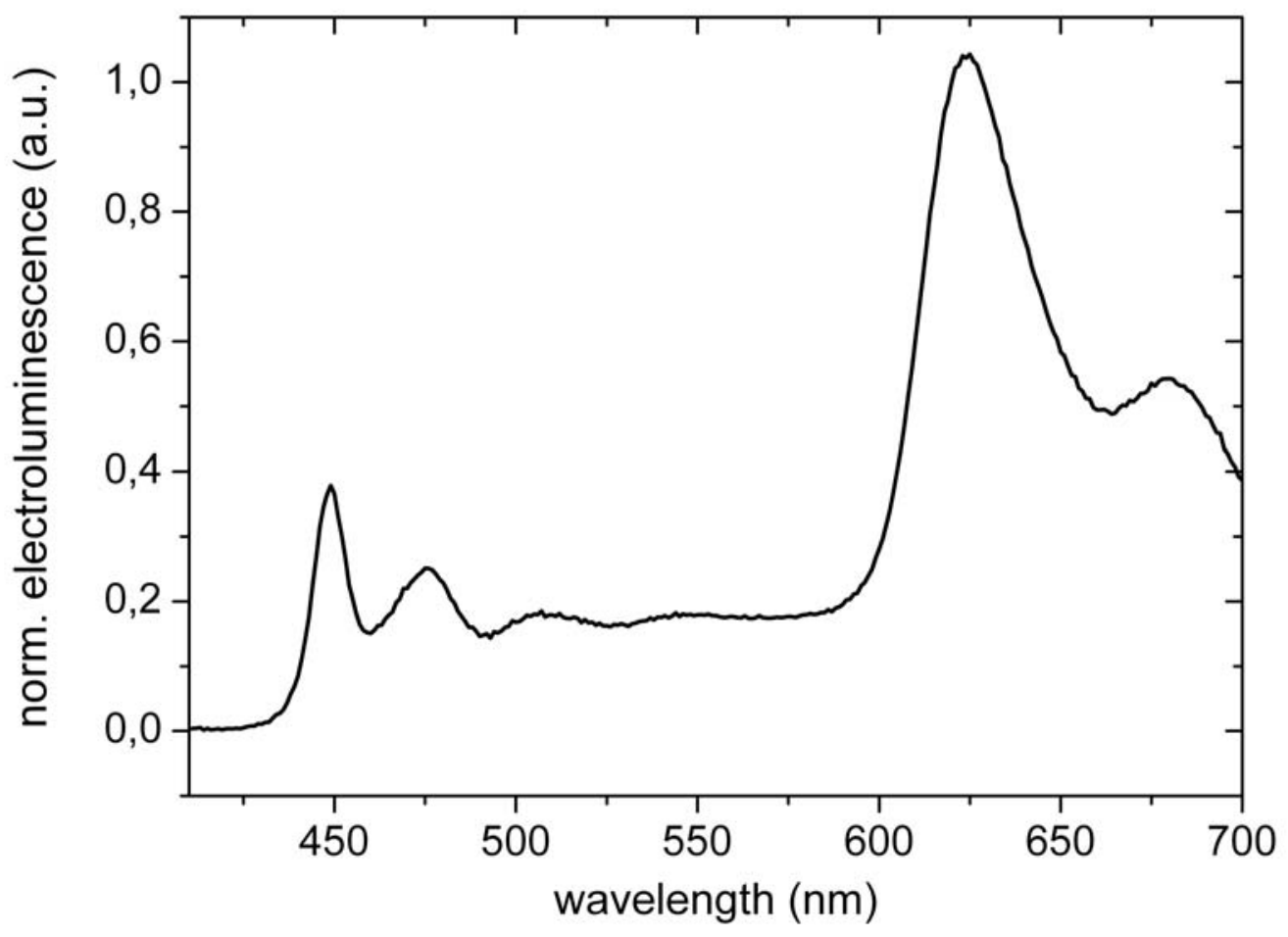

Figure S18. Normalized electroluminescence spectrum of 3 in LPPP

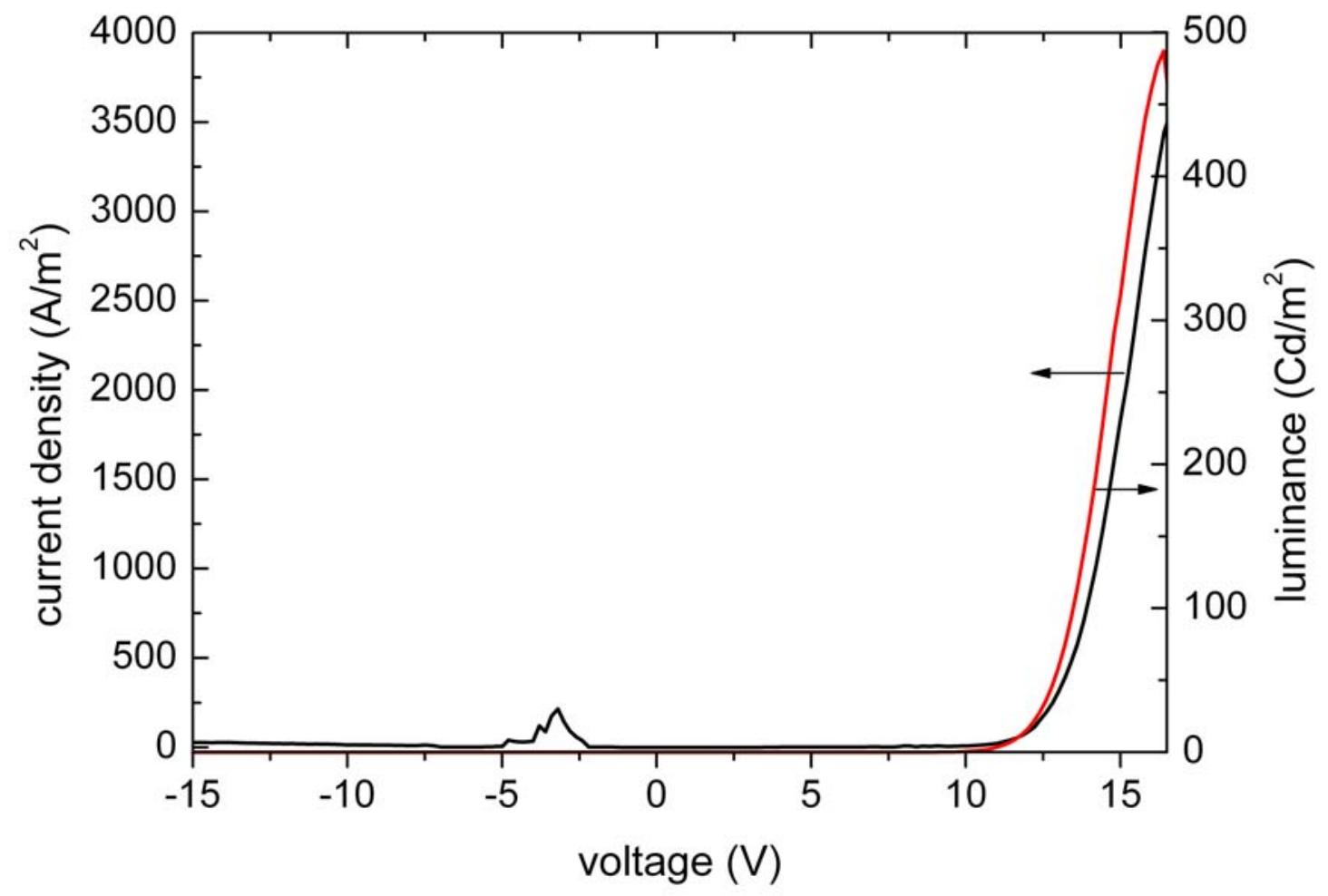

Figure S19. Bias/current characteristics and bias/electroluminescence characteristics of 3 in LPPP 


\section{Stability in the Presence of conc. TFA}

To test the stability of $\mathbf{1}$ in the presence of conc. TFA, a $5 \mathrm{mg}$ sample of $\mathbf{1}$ was dissolved in $\mathrm{CDCl}_{3}$. After measuring the corresponding ${ }^{1} \mathrm{H}-\mathrm{NMR}$ spectrum, $20 \mu \mathrm{L}$ of conc. TFA (spectrophotometric grade) were added to the same sample and the mixture was stirred for 1 hour at room temperature. The solvent was removed in vacuo and the residue was dried for 24 hours. After re-dissolving of the sample in $\mathrm{CDCl}_{3}$, the corresponding ${ }^{1} \mathrm{H}-\mathrm{NMR}$ spectrum of $\mathbf{1}$ after exposure to conc. TFA was recorded.

As depicted in Figure S19, the exposure to conc. TFA causes only slight differences of the ${ }^{1}$ H-NMR spectra. Although some differences can be noted (presumably a result of the changed solvent polarity by the presence of residual TFA), characteristic NMR-signals such as $\mathrm{py}^{6}$ or $\mathrm{ph}^{3}$ remain virtually unchanged.

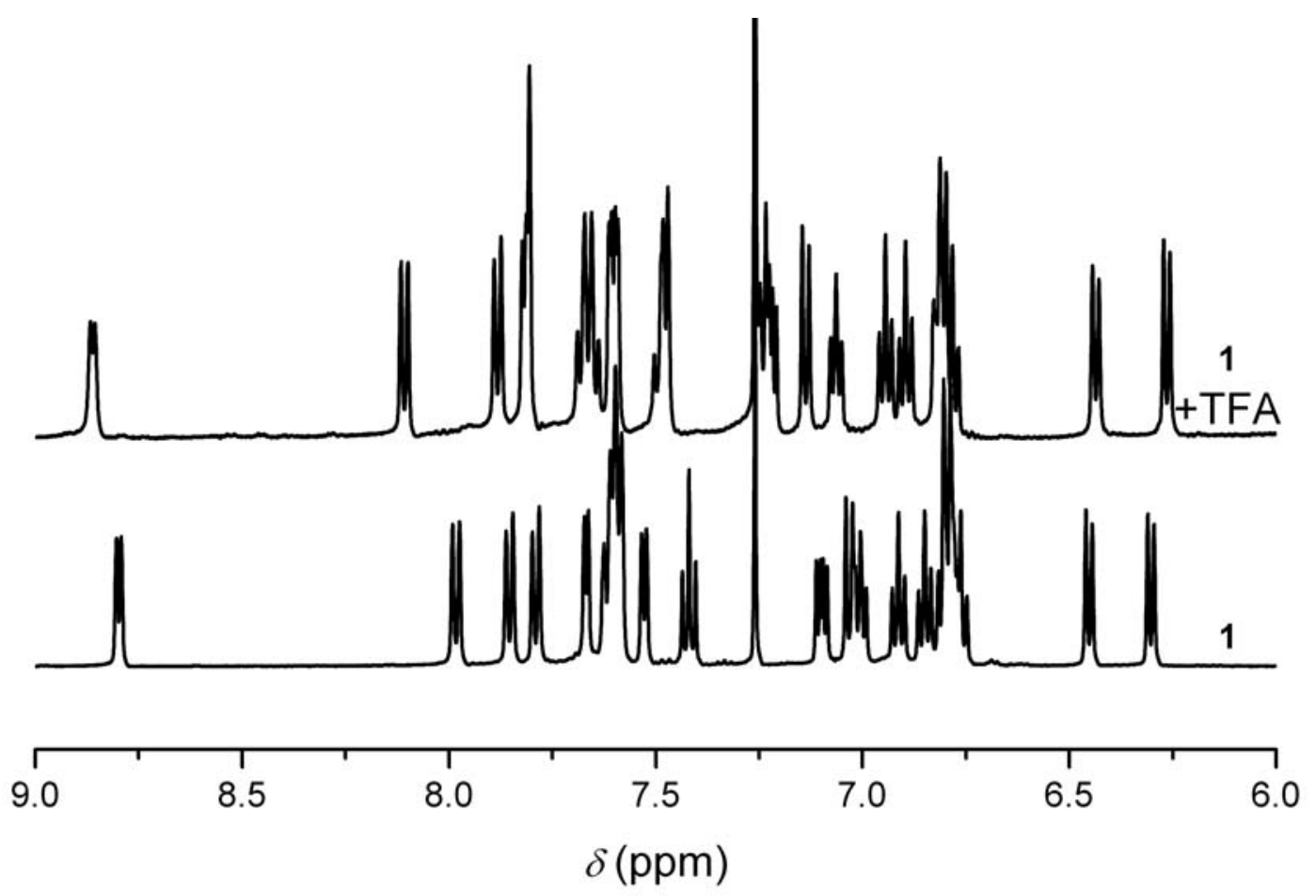

Figure S20. ${ }^{1} \mathrm{H}-\mathrm{NMR}$ spectra of $\mathbf{1}$ in $\mathrm{CDCl}_{3}$ before and after exposure to conc. TFA 